\title{
Avaliação da Comunicabilidade da WABlind em Enciclopédias On-line
}

\author{
Natacsha Raposo $^{1}$, Thais Castro ${ }^{1}$, Alberto Castro ${ }^{1}$ \\ ${ }^{1}$ Instituto de Computação - Universidade Federal do Amazonas (UFAM) \\ Manaus - AM - Brazil \\ \{natacsha, thais, alberto\} @icomp.ufam.edu.br
}

\begin{abstract}
People with disabilities study, use the internet and other TDIC features using Assistive Technologies (TA). Thus, it is necessary to reflect on content customizations in an educational environment, carried out by the teacher. In particular, the use of the web by visually impaired people is supported by TA screen readers. However, while such readers synthesize information from this content, people with visual impairment may find difficulties in understanding it due to the lack of necessary resources, applied at the stage of its construction. Therefore, this article describes an experimental evaluation using the WABlind tool with the mediation of teachers to configure the necessary adaptations to their students. Test results suggest that the tool is an aid in content customizations for visually impaired people.
\end{abstract}

Resumo. Pessoas com deficiências estudam, utilizam a internet e outros recursos de TDIC usando tecnologias assistivas (TA). Dessa forma, faz-se necessário refletir sobre personalizações de conteúdo em ambiente educacional, realizado pelo professor. Em especial, o uso de web por pessoas com deficiência visual é apoiado por TA leitores de tela. No entanto, embora tais leitores sintetizem a informação deste conteúdo, pessoas com deficiência visual podem encontrar dificuldades em sua compreensão devido à ausência de recursos necessários, aplicados na etapa da sua construção. Diante disso, este artigo descreve uma avaliação experimental utilizando a ferramenta WABlind com a mediação dos professores para configurar as adaptações necessárias aos seus alunos. Os resultados dos testes sugerem que a ferramenta é um auxílio nas personalizações de conteúdo a pessoas com deficiência visual.

\section{Introdução}

Segundo Censo (2010), no Brasil existem aproximadamente 46 milhões de pessoas com deficiência (PCDs), ou cerca de $24 \%$ da população total. Entre os tipos de deficiência, a mais declarada foi a deficiência visual. De acordo com o documento expedido pelo IBGE (2010) - Características Gerais da População, Religião e Pessoas com Deficiência - cerca de 35,8 milhões de pessoas relataram ter deficiência visual grave, muito difícil ou sem capacidade de ver. Pessoas com deficiências visuais têm acesso ao conteúdo web por meio de tecnologias assistivas, especificamente por leitores de tela. As Tecnologias Assistivas promovem o Decreto $n^{\circ} 5.296$ de dezembro/2004, que regulamenta a acessibilidade deve ser fornecida a todos ou o maior número possível de usuários em situações limitantes [WCAG, 2014]. Essas tecnologias destinam-se a 
VII Congresso Brasileiro de Informática na Educação (CBIE 2018)

Anais do XXIX Simpósio Brasileiro de Informática na Educação (SBIE 2018)

proporcionar maior independência às pessoas com deficiência, de modo que seu dia a dia se torne mais simplificado com ferramentas ou utilidades [WAI, 2014].

A Lei brasileira de inclusão, n. 13.146 (2015) trata sobre a inclusão da pessoa com deficiência em seus diversos contextos, inclusive o escolar. Especificamente, em seu artigo 28, parágrafo XII são descritos os tipos de mecanismos que as escolas devem fornecer: "oferta de ensino de Libras, do Sistema Braille e de uso de recursos de tecnologia assistiva, de forma a ampliar habilidades funcionais dos estudantes, promovendo sua autonomia e participação". No caso de pessoas com deficiência visual, por exemplo, somente o sistema braille não a fará ser incluída em ensino regular, precisando de tecnologias assistivas, como adaptações de conteúdo web, por exemplo. Essas adaptações são necessárias pois no cotidiano escolar atual, os alunos precisam consultar frequentemente sites de enciclopédias educacionais, além de vídeos e slides, para terem acesso ao conteúdo escolar necessário à realização das atividades.

Diante disso, maximizar o acesso às informações disponíveis no conteúdo da web aparece como um fator importante na vida dos deficientes visuais, uma vez que possibilitar que elementos originalmente inacessíveis se tornem acessíveis amplia o escopo das informações a serem consumidas. Nessa perspectiva, as ferramentas web apresentam-se promissoras na tentativa de ampliar as possibilidades de inclusão social e melhorar a qualidade de vida das pessoas que possuem algum tipo de limitação, seja ela circunstancial ou permanente.

Nesse contexto, este trabalho tem o objetivo de apresentar a ferramenta WABlind, uma ferramenta web que aplica diretrizes do WCAG 2.0 e trata itens problemáticos, apontados pelo $\mathrm{W} 3 \mathrm{C}$, ocorridos na interação de conteúdo da web por pessoas com deficiência visual, e o resultado do teste experimental realizado com professores para sintonização de personalizações e adaptações de sites de enciclopédias escolares, os quais gostariam que seus alunos tivessem acesso para realizar suas pesquisas relativas às suas disciplinas.

\section{Fundamentação Teórica}

Segundo [Silva et al., 2005] a inclusão digital é parte do fenômeno informação, no contexto da chamada sociedade da informação que pode ser observada pela ótica da ciência da informação sob o aspecto de ser considerada como ponto de partida e como ponto de chegada. Onde o ponto de partida é o acesso à informação, que está nos meios digitais e, o ponto de chegada, a assimilação da informação e sua reelaboração em novo conhecimento, a fim de possibilitar a melhoria da qualidade de vida das pessoas.

Trabalhos que visam avaliar e promover a inclusão de pessoas em ambientes de aprendizagem por meio de objetos educacionais apresentam resultados satisfatórios sobre a utilização de conteúdo digital como objeto educacional, quando direcionados por professores [Costa et al. 2003], [Almeida 2003], [Oliveira 2017]. No caso de sites educacionais utilizados como objeto de aprendizagem é importante refletir sobre os elementos expostos nesses sites, onde muitas vezes eles não estão relacionados ao conteúdo principal, por exemplo banners e links, e o professor não possui privilégios de reorganização de tal conteúdo.

Acessibilidade na Web significa que pessoas com deficiências podem usar a Web [W3C, 2005]. Para alcançar a acessibilidade na Web, o W3C criou em 1999 a WAI (Web Accessibility Initiative), formada por grupos de trabalho focados no desenvolvimento de diretrizes, denominadas Diretrizes de Acessibilidade para Conteúdo da Web - WCAG. O WCAG 2.0 inclui uma série de recomendações para tornar o conteúdo da web mais acessível. Seguir essas recomendações pode tornar o conteúdo acessível a um amplo grupo de pessoas com deficiência, incluindo cegueira e baixa visão, surdez e baixa audição, dificuldades de aprendizagem, limitações cognitivas, movimentos limitados, deficiências de fala e outras deficiências [WCAG, 2014]. 
VII Congresso Brasileiro de Informática na Educação (CBIE 2018)

Anais do XXIX Simpósio Brasileiro de Informática na Educação (SBIE 2018)

A literatura voltada para acessibilidade na web é muito ampla e abrange muitas deficiências, como déficit cognitivo, incapacidade física, como por exemplo, motora, auditiva, visual, entre outras. Neste trabalho, listamos alguns trabalhos que discutem problemas de manipulação de conteúdo da web para disponibilizá-lo em uma abordagem mais acessível aos usuários em geral. Na tentativa de minimizar a falta de mobilidade na navegação de conteúdo da web por pessoas com deficiência visual, podese citar o WebAnywhere: O leitor de tela em movimento [Bigham et al., 2008]. A ferramenta web não precisa ser baixada ou instalada: o WebAnywhere acessa o texto em um servidor externo e envia o arquivo de áudio para reprodução em seu navegador. Assim, o texto sintetizado pode ser executado em qualquer computador com altofalantes ou fones de ouvido. Assim, onde quer que estejam os deficientes visuais, podem acessar o conteúdo da web, navegar e ouvir as instruções.

Como mostrado no trabalho Automatic Reverse Engineering of Interactive Dynamic Web Applications to Support Adaptation across Platforms [Bellucci et al., 2012] é possível fazer engenharia reversa de páginas dinâmicas. Em particular, esse trabalho discute como a ferramenta de engenharia reversa permite a adaptação dinâmica das páginas que os usuários podem acessar através de vários dispositivos. Há ainda outros projetos como os colaborativos, também com a finalidade de permitir informação acessível [BE MY EYES, 2018]. Além das possibilidades de adaptação do conteúdo da web, a representação descritiva dos seus elementos se faz essencial para ampliar o alcance da informação acessada. Trabalhos como os descritos em [Manola; Miller, 2004], [Mey, 1995], [Nilsson et al., 2008] apontam que este tipo de representação tem sido buscado para diferentes tipos de conteúdo.

Considerando as desvantagens atuais das ferramentas existentes para melhorar a acessibilidade de conteúdo da web, foi desenvolvida a WABlind, apresentada na próxima seção, que visa o apoio à identificação de elementos de conteúdo da web, com inclusão de marcadores, visando manter os elementos importantes e assim não descaracterizar a originalidade desse conteúdo ao adaptá-lo aos leitores de tela, partindo de uma representação descritiva colaborativa (por mediadores) desses elementos.

\section{WABlind - Web Accessibility for Blind People}

A ferramenta WABlind foi desenvolvida para tratar diferentes níveis de acessibilidade em conteúdo da web. Atualmente são dois os níveis implementados: o primeiro trata problemas sintáticos, classificados conforme o WCAG 2.0 como nível A e o segundo auxilia na marcação de elementos do conteúdo da web, possibilitando alternativas de áudio para que o leitor o sintetize.

Para validação do primeiro nível foram realizados testes de conformidade com o validador AChecker [ACHECKER, 2011]. As diretrizes tratadas e os resultados dos testes realizados com 500 páginas mais acessadas (de acordo com http:// www.alexa.com) estão no Relatório Técnico de Teste de Conformidade da Ferramenta WABlind [Raposo, O. N., Castro, T. \& Castro, A., 2018].

Na segunda fase, o nível específico é realizado pela associação de marcadores predefinidos para elementos do conteúdo da web por meio de uma url fornecida. Essa associação é construída de forma colaborativa, onde o usuário previamente cadastrado no ambiente tem acesso a uma área restrita. Os marcadores foram definidos com base em elementos comumente encontrados em conteúdo da web, como a página do menu principal, a área da caixa de pesquisa e outros (por exemplo, imagens, vídeos) que possuem informações visuais e exigem uma descrição alternativa. Esses marcadores têm como objetivo representar descritivamente elementos de conteúdo da web. O conteúdo da web a ser carregado nesse nível é pré-processado pelos mecanismos de processamento de níveis automatizados. Logo, quando a conteúdo está disponível para cadastro da descrição dos elementos ele já foi previamente tratado no nível 1. A Figura 1 apresenta o fluxo da WABlind para tratamento do conteúdo da web. 
VII Congresso Brasileiro de Informática na Educação (CBIE 2018)

Anais do XXIX Simpósio Brasileiro de Informática na Educação (SBIE 2018)

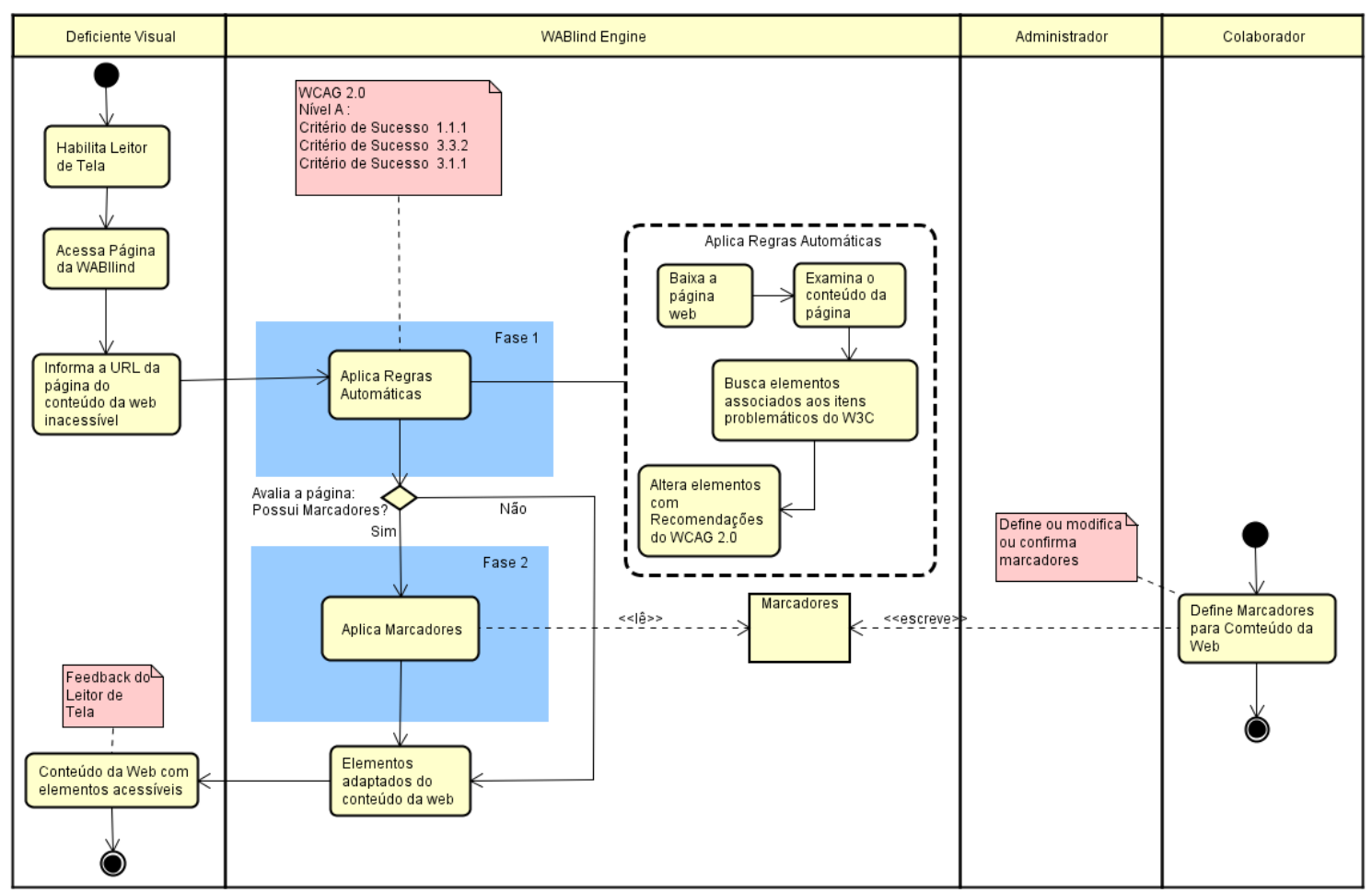

Figura 1. Esquema das atividades realizadas no tratamento de uma página web pela ferramenta WABlind.

\section{Avaliação Experimental}

Este trabalho foi planejado com base em uma avaliação experimental incluindo uma análise qualitativa. Essa análise teve como objetivo a rotulação de elementos de um site de enciclopédia de conhecimentos gerais por meio da associação de marcadores da ferramenta WABlind.

Foram analisados trabalhos relacionados ao desenvolvimento de conteúdo acessível e de representação descritiva de conteúdo da web que possibilitaram integrar novos conhecimentos para a elaboração da avaliação realizada. Os dados coletados foram analisados qualitativamente utilizando o Método de Avaliação da Comunicabilidade (MAC) [Prates, de Souza, C. S., \& Barbosa 2000]. Esse método simula a comunicação do usuário com o projetista sobre a interface, que tem como base a teoria da Engenharia Semiótica de Souza e tem como objetivo avaliar a recepção da mensagem pelos usuários a fim de analisar a qualidade desta recepção (2005).

\subsection{Materiais e Métodos}

O teste foi realizado com 12 participantes. Dentre eles haviam professores e especialistas em comunicabilidade com faixa etária entre 25 e 59 anos. Os critérios para selecionar professores para o experimento foi sua experiência prévia em lecionar ao menos uma disciplina para alunos com algum tipo de deficiência, incluindo deficientes visuais e que fossem hábeis no uso das TDIC.

$\mathrm{O}$ experimento foi realizado em duas fases. Na primeira foram realizados testes de inspeção de usabilidade a fim de analisar se as interfaces da ferramenta apresentavam problemas que pudessem prejudicar seu uso adequado. A segunda teve como objetivo avaliar a utilização dos marcadores de representação descritiva dos elementos do conteúdo da web. Dos 12 participantes, 7 eram, além de professores, especialistas em usabilidade e comunicabilidade e 5 eram professores com experiência. A interação em 
VII Congresso Brasileiro de Informática na Educação (CBIE 2018)

Anais do XXIX Simpósio Brasileiro de Informática na Educação (SBIE 2018)

ambas foi gravada a fim de identificar possíveis rupturas não mencionadas nos questionários.

$\mathrm{O}$ acesso a ferramenta WABlind pelos participantes foi guiado por meio de um roteiro textual e um áudio explicando o propósito da ferramenta. A execução de um roteiro único em ambas as fases teve como propósito coletar e observar diferentes variáveis em um mesmo contexto. Por fim, foi realizada a avaliação da interface com relação à qualidade da comunicação do projetista para com os usuários, utilizando o MAC [Prates, de Souza, C. S., \& Barbosa 2000].

\subsection{Método de Avaliação da Comunicabilidade - MAC}

Em um teste de comunicabilidade é necessária a gravação da interação do usuário com o sistema durante todo o processo, além de anotações feitas pelos avaliadores, pois posteriormente será feita uma análise dos registros coletados. Segundo Prates e Barbosa (2003), o método deve gerar:

1. Uma etiquetagem, que consiste em assistir as gravações da interação e atribuir a expressão apropriada nos momentos de ruptura da interação;

2. Uma interpretação, que consiste em tabular e consolidar a informação obtida, ou seja, as expressões obtidas, associando-as a classificações de problemas de interação ou diretrizes de design;

3. E, um perfil semiótico, que consiste em interpretar a tabela resultante do passo anterior, dentro do quadro teórico da Engenharia Semiótica, em uma tentativa de se reconstruir a metamensagem sendo transmitida pelo projetista ao usuário através da interface [de Souza, 1991] [de Souza, 2003].

\subsubsection{Preparação}

Os testes para essa pesquisa foram realizados com 12 participantes. Quanto à escolaridade, dos 12 participantes 4 eram doutores, 2 especialistas e 7 mestres. Dentre os 12 participantes 8 possuíam experiência ministrando aula para alunos com deficiência visual, 2 com deficiência intelectual, $7 \mathrm{com}$ deficiência auditiva e $1 \mathrm{com}$ surdocego. O experimento ocorreu do mesmo modo, com cada participante, na respectiva ordem de passos: um cenário contendo as tarefas a serem executadas, $\mathrm{o}$ preenchimento do TCLE e do Questionário do Perfil.

O primeiro questionário possuía 7 questões. Tal questionário tinha como objetivo coletar informações sobre o nível de formação, experiência profissional na área e se já haviam atuado com pessoas com algum tipo de deficiência. O segundo questionário possuía 9 questões objetivas relacionadas à experiência do uso da ferramenta. Após preenchimento do Questionário de Perfil, o participante acessava a ferramenta WABlind, disponível no link http://wablind.icomp.ufam.edu.br, para execução do roteiro. Durante a execução do roteiro a interação com a ferramenta foi gravada. Com a finalização das tarefas o participante fazia o preenchimento do segundo questionário.

O experimento teve como objetivo identificar a viabilidade da ferramenta WABlind sobre dois aspectos a) Interação com a Ferramenta para representar descritivamente elementos de conteúdo da web b) Disponibilidade dos tipos de marcadores para representar descritivamente elementos de conteúdo da web.

Para efetuar a coleta dos dados foram realizadas duas fases seguindo a ordem respectiva dos aspectos mencionados anteriormente. A primeira fase foi realizada pelos 7 professores especialistas em comunicabilidade e usabilidade. A segunda pelos outros 5 professores. Após a primeira fase, a ferramenta foi ajustada conforme feedback da primeira fase. Cada fase foi dividida em 3 etapas: a) Questionário de Identificação do 
VII Congresso Brasileiro de Informática na Educação (CBIE 2018)

Anais do XXIX Simpósio Brasileiro de Informática na Educação (SBIE 2018)

Perfil b) Experimentação da Ferramenta c) Questionário Pós Uso da Ferramenta. Na segunda fase foram avaliados os marcadores que estavam disponíveis na ferramenta.

\subsubsection{Coleta de Dados}

Os avaliadores de comunicabilidade foram uma especialista em Engenharia Semiótica e 2 alunos de doutorado do Programa de Pós-Graduação da XXXXX, com experiência em métodos de avaliação da Engenharia Semiótica.

Mediante as características do método, todos os testes foram gravados por um software instalado na máquina onde a aplicação foi acessada. Todos os participantes consentiram com as filmagens que seguiram os aspectos éticos conforme lei (Brasil, 1996). O TCLE foi lido pelo avaliador para o participante, e este assinou no local indicado.

\subsubsection{Análise de Dados}

Nesta fase é realizada a etiquetagem que caracterizam o tipo de ruptura de comunicação entre usuário e sistema (preposto do projetista). Após isso é realizada a interpretação da etiquetagem, onde o avaliador tabula os problemas identificados e sua interpretação, que depende da sua experiência e conhecimento em Engenharia Semiótica.

Alguns aspectos devem ser considerados durante a interpretação para permitir ao avaliador identificar os principais problemas da metacomunicação: (1) classificação das expressões que caracterizam a ruptura quanto ao tipo de falha que representam na comunicação entre o sistema (enquanto preposto do designer) e usuário; (2) a frequência e contexto em que ocorrem as rupturas; (3) identificação de padrões de sequências de expressões; (4) o nível da ação em que ocorre a ruptura.

Os tipos de falha são definidos em função da relação entre a intenção de uma comunicação e o efeito que ela causa. Estas falhas podem ser definidas como completas, parciais ou temporárias [Prates, 2007]: falhas completas acontecem quando a intenção da comunicação e seu efeito são inconsistentes; falhas parciais quando parte do efeito pretendido da comunicação não é atingido; e falhas temporárias que ocorrem na expressão ou intenção de um ato comunicativo entre usuário e sistema, e que são percebidas pelo usuário que tenta então superá-las.

Tabela 1. Etiquetagem de rupturas de interação do sistema - Fase 1.

\begin{tabular}{|l|c|c|c|c|c|c|c|}
\hline \multirow{2}{*}{\multicolumn{1}{c|}{ Etiquetas }} & \multicolumn{7}{c|}{ Professor } \\
\cline { 2 - 9 } & 1 & 2 & 3 & 4 & 5 & 6 & 7 \\
\hline Desisto & & & & & & & \\
\hline Pra mim, está bom. & & & 1 & 1 & 1 & 1 & 1 \\
\hline Não, obrigado. & & & & & & & \\
\hline Vai de outro jeito. & 1 & 1 & 1 & & 1 & & \\
\hline Cadê? & 2 & 2 & 1 & 1 & 1 & & \\
\hline Ué, o que houve? & 1 & & & & & & \\
\hline E agora? & 1 & & & & 1 & & \\
\hline Onde estou? & & & & & & & \\
\hline Epa! & & & 1 & & & & \\
\hline Assim não dá. & & & & & & & \\
\hline O que é isto? & & 1 & & & & & \\
\hline Socorro & & & & & & & \\
\hline Por que não funciona? & & 1 & & & & & \\
\hline
\end{tabular}


VII Congresso Brasileiro de Informática na Educação (CBIE 2018)

Anais do XXIX Simpósio Brasileiro de Informática na Educação (SBIE 2018)

Diante disso, inicialmente cada avaliador realizou o processo de etiquetagem e suas respectivas interpretações, individualmente; e, em seguida foi realizado um debate para discutirem-se as rupturas encontradas. Após isso, foi gerada uma etiquetagem final da análise das filmagens, conforme consenso das rupturas que ocorreram na comunicação com o sistema. A Tabela 1 mostra a frequência das rupturas.

A partir desse ponto, seguindo a sequência definida previamente, ocorreu o processo de interpretação semiótica das rupturas ocorridas nas interações de cada participante. A seguir, as principais rupturas encontradas na avaliação:

1. Participante não consegue perceber que o elemento da página está selecionado e continua a navegar na página.

Tipo de falha: Temporárias

Etiqueta (s): "Ué o que houve?"

Descrição/Justificativa: $O$ participante encontrou dificuldades para conseguir identificar a sinalização do elemento selecionado para fazer a associação do marcador.

2. Participante não encontra um marcador adequado para associar ao elemento.

Tipo de falha: Temporárias

Etiqueta (s): "Cadê?"

Descrição/Justificativa: O participante fica a procurar algum marcador do sistema que se adeque a sua necessidade.

3. Participante acreditava que estava criando elementos marcados apenas clicando sobre eles. Sem ter que associar o marcador ao elemento.

Tipo de falha: Completas

Etiqueta (s): "Pra mim está bom".

Descrição/Justificativa: O sistema habilitava a opção de marcadores com o botão direito do mouse. Quando se clicava com o botão direito apareciam várias opções do browser, além da opção dos marcadores.

Antes da execução da segunda fase do teste foram realizados reparos na ferramenta conforme rupturas apontadas na fase 1. Na segunda fase foi realizada uma análise qualitativa sobre o feedback dos participantes e sobre a disponibilidade de marcadores. Dos 5 participantes 2 afirmaram sentir a necessidade de outros marcadores na ferramenta. Sugeriram que houvesse marcadores para autores do conteúdo, exercícios extras relacionados e links de redes sociais.

\subsubsection{Fechamento e Discussão}

Dos 12 testes realizados todos foram finalizados. O alto número de rupturas de comunicação completas e temporárias nos permite considerar que elas se devem a problemas de comunicabilidade relacionados aos mecanismos de interação do sistema para as funcionalidades especificadas nas tarefas. No geral, a dificuldade da interação se deu porque os participantes não estavam acostumados com a tarefa de associar elementos em uma página web. Sentiram, portanto, dificuldades para elencar marcadores de páginas conforme achassem adequados à sua linguagem.

A última atividade dessa avaliação é a consolidação da metamensagem projetista-usuário. É importante observar que essa atividade de inspeção realizada, conduzida pelos resultados das fases de observação de usuários, etiquetagem e interpretação, é o que diferencia o MAC de um teste de usabilidade [CommEST, 2007]. 
VII Congresso Brasileiro de Informática na Educação (CBIE 2018)

Anais do XXIX Simpósio Brasileiro de Informática na Educação (SBIE 2018)

Em seguida, partimos então para o desfecho do estudo que consistiu na geração do perfil semiótico, onde reconstruímos a metamensagem do projetista-usuário.

[Aqui está o meu entendimento sobre quem você é]. Você é um docente que deseja compartilhar materiais de apoio da disciplina com a turma que costuma indicar sites educacionais para seus alunos e possui conhecimento sobre o que é relevante em sites como esses. [O que eu aprendi que você quer, ou precisa fazer, de que forma e porque]. Você precisa que a ferramenta sinalize os elementos que você selecionou de forma diferenciada conforme os marcadores que você associou. Após a associação dos marcadores você gostaria de poder excluir os marcadores e que fosse sinalizado quais são as suas contribuições dentre eles. [Este é o aplicativo que eu projetei para você e é essa a maneira pela qual você pode ou deve utilizá-lo de forma que preencha uma variedade de propósitos que coordenam com essa visão]. Você pode acessar a ferramenta com usuário e senha previamente cadastrado, pode realizar o acesso também com a conta do Facebook. Você terá acesso a uma página explicando o significado de cada marcador e um exemplo de um conteúdo relacionado. A partir disso, você poderá fazer a associação dos marcadores de modo colaborativo.

Conforme evidenciado no perfil semiótico, partindo da análise das rupturas, durante o experimento algumas dificuldades foram encontradas pelos participantes na realização de suas tarefas. Na fase 1 os participantes relataram dificuldades para fazer a marcação da página devido ao modo como foi projetada a associação dos marcadores. $\mathrm{Na}$ fase 2 pôde-se perceber que os professores não possuíam conhecimento aprofundado sobre estruturas de sites de enciclopédia, somente sabendo que possuem conteúdo desejado, o que dificulta a associação dos marcadores, pois não se tem a percepção do que é conteúdo relevante.

Diante da análise feita na avaliação propomos as seguintes sugestões: visto que a tarefa mais crítica foi a de acesso aos marcadores, uma opção seria apresentar uma visão geral sobre as categorias de marcadores e seus subitens.

\section{Considerações Finais}

Apesar da semelhança do WABlind com os trabalhos apresentados anteriormente, existem algumas diferenças significativas. O conteúdo acessível gerado por [Bellucci et al., 2012], possui a ausência de mecanismos de validação da sua acessibilidade. A adaptações geradas pelo trabalho de engenharia reversa automática de aplicações interativas da Web dinâmica para apoiar a adaptação entre plataformas não tem como objetivo adequar as informações dispostas e sim a estrutura da página.

Existem diversos cenários nos quais as pessoas com deficiência encontram dificuldades ao acessar um conteúdo da web. E importante observar que existem diversos casos que podem ser analisados como o de acentuação, pois se o caractere não for convertido, dependendo do charset definido da página a leitura da informação não ficará nítida; outro caso é o de imagens que não possuem um texto alternativo: neste caso o usuário deveria ser notificado que naquela área existe uma imagem que não pode ser traduzida, ou até mesmo ter a opção de ignorar mídias.

Os testes realizados apontam que a ferramenta segue as diretivas da acessibilidade, tendo passado nos testes de conformidade previamente realizados, e ainda que sua camada de personalização das diretivas de adaptação é útil e consistente para ser utilizada por mediadores acadêmicos, como os professores, necessitando somente de alguns ajustes em sua interface para simplificar a linguagem textual utilizada com os mediadores na personalização.

O diferencial desta ferramenta são as tratativas aplicadas aos elementos, originalmente, inacessíveis, do conteúdo da web, o que possibilita o fornecimento de alternativa textual para que os leitores de tela possam sintetiza-los durante a navegação. Além dessas tratativas, a WaBlind possui uma camada de personalização de conteúdo 
VII Congresso Brasileiro de Informática na Educação (CBIE 2018)

Anais do XXIX Simpósio Brasileiro de Informática na Educação (SBIE 2018)

que pode ser utilizada pelos próprios professores para personalizarem as adaptações conforme seus objetivos pedagógicos.

\section{Referências}

ACHECKER. Web Accessibility Checker, 2011. Disponível em: http://achecker.ca/checker/index.php. Acesso em: 03 nov 2017.

Almeida, Maria Elizabeth Bianconcini de. Educação a distância na internet: abordagens e contribuições dos ambientes digitais de aprendizagem. Educ. Pesqui. [online]. (2003), vol.29, n.2, pp.327-340. ISSN 1517-9702. http://dx.doi.org/10.1590/S151797022003000200010

Barbosa, S.D.J. and Silva, B.S. da. Interação Humano-Computador. Elsevier Editora Ltda., (2010).

Be My Eyes - Bringing sight to blind and people with low vision. Disponível em: https://www.bemyeyes.com/. Acesso em: 25 jan 2018.

Bellucci, F., Ghiani, G., Paternò, F., Porta, C.: Automatic Reverse Engineering of Interactive Dynamic Web Applications to Support Adaptation across Platforms. In: Proc. IUI 2012, PP. 217-226. ACM (2012)

Brasil. Ministério Nacional da Saúde. Conselho Nacional de Saúde. Resolução 196/96 sobre pesquisa envolvendo seres humanos. Journal of the Electrochemical Society, 1996.

CommEST - Uma ferramenta de apoio ao método de Avaliação de Comunicabilidade / Luciana Cardoso de Castro Salgado ; orientador: Clarisse Sieckenius de Souza. 2007.

Costa, Valéria Machado., Rapkiewicz, Clevi., Filho, Mário Galvão., Canela, Maria. Avaliação de sites educacionais de Química e Física: um estudo comparativo. (2003). Disponível em: http://brie.org/pub/index.php/wie/article/download/820/806. Acesso em: 05 fev 2018.

de Souza, C. S. (1993), The Semiotic Engineering of User Interface Languages, International Journal of Man-Machine Studies, v. 39, pp. 753-773.

de Souza, C. S., Barbosa, S. D. J., and Prates, R. O. (2001), A Semiotic Engineering Approach to User Interface Design. Knowledge Based Systems. Amsterdam, v. 14, n. 8, pp. 461-465.

de Souza, C. S. (2005) The Semiotic Engineering of Human-Computer Interaction. Cambridge, MA: The MIT Press.

IBGE, "Características Gerais da População, Religião e Pessoas com Deficiência: Publicação Completa,". (2010). Disponível: http://servicodados.ibge.gov.br/Download/Download .ashx?http=1\&u=biblioteca.ibge .gov.br/visualizacao/periodicos/94/cd_2010_religiao_deficiencia.pdf. Acesso em: 20 fev 2018.

IBGE, "Sinopse do Censo Demográfico 2010,". (2010). Disponível: http://www.ibge.gov.br/home/estatistica/populacao/censo2010/default_sinopse.shtm. Acesso em: 20 fevereiro 2018.

J. P. Bigham, C. M. Prince and R. E. Ladner, "WebAnywhere: A Screen Reader On-theGo," In Proceedings of the 2nd Cross-Disciplinary Conference on Web Accessibility, (2008).

Manola, F.; Miller, E. Rdf Primer. (2004). Disponível em: http://www.w3.org/TR/2004/REC-rdf-primer-20040210/. Acesso em: 1 mar 2018. 
VII Congresso Brasileiro de Informática na Educação (CBIE 2018)

Anais do XXIX Simpósio Brasileiro de Informática na Educação (SBIE 2018)

Mey, E.S.A. Introdução à catalogação. Brasília: Briquet de Le-mos, (1995).

Nilsson, M. et al. Expressing Dublin Core metadata using the resource description framework. Singapore: DCMI, (2008). Disponível em: http://dublincore.org/documents/dc-rdf. Acesso em: 14 mai 2018.

Oliveira, Flávia Medianeira de. O USO DE PORTAIS EDUCACIONAIS NO ENSINO-APRENDIZAGEM DE INGLÊS PARA FINS ESPECÍFICOS. Alfa, rev. linguíst. (São José Rio Preto) [online]. (2017), vol.61, n.3, pp.653-671. ISSN 00025216. http://dx.doi.org/10.1590/1981-5794-1711-8.

Prates, R. O., de Souza, C. S., \& Barbosa, S. D. (2000). Methods and tools: a method for evaluating the communicability of user interfaces. interactions, 7(1), 31-38.

Prates, R. O.; Barbosa, Simone Diniz Junqueira. Avaliação de Interfaces de Usuário Conceitos e Métodos (2003). In: Juan Manuel Adán Coello; Sandra C. P. Ferraz Fabbri. (Org.). Jornada de Atualização em Informática do Congresso da Sociedade Brasileira de Computação. Campinas: SBC, 2003, v. 2, p. 245-293.

Prates, R. O. \& Barbosa, S.D.J. Introdução à Teoria e Prática da Interação HumanoComputador fundamentada na Engenharia Semiótica. (2007). In T. Kowaltowski \& K. Breitman (orgs.) Jornadas de Atualização em Informática, JAI 2007, pp. 263-326.

Raposo, N., Rios, H., Lima, D., Gadelha, B. \& Castro, T. (2014). An application of mobility aids for the visually impaired.. In A. B. Zaslavsky, S. W. Loke, L. Kulik \& E. Pitoura (eds.), MUM (p./pp. 180-189), : ACM. ISBN: 978-1-4503-3304-7

Raposo, O. N., Castro, T., Castro, A. (2018) RT-GSI-2018-004. Teste de Conformidade da Ferramenta WABlind. Relatório Técnico do Grupo de Pesquisa de Sistemas Inteligentes - GSI.

Silva, Helena; JAMBEIRO, Othon; LIMA, Jussara and BRANDAO, Marco Antônio. Inclusão digital e educação para a competência informacional: uma questão de ética e cidadania. Ci. Inf. [online]. (2005), vol.34, n.1, pp.28-36. ISSN 0100-1965. http://dx.doi.org/10.1590/S0100-19652005000100004.

Souza, Z. M.; Leal, G. C. L.; Huzita, E. H. M., (2012). Um exemplo de condução de estudo experimental guiado por um processo; Revista Tecnológica (UEM), Maringá - PR, V.21, pg 43 - 52, ISSN: 1517-8048, Meio digital, http://periodicos.uem.br/ojs/index.php/RevTecnol/article/view/9928/1027

W3C, "Acessibilidade para o WAI," (2005). Disponível em: http://www.w3.org/WAI/intro/accessibility.php. Acesso em: 20 abr 2018.

W3C, "Resultados preliminares - Pesquisa sobre uso de Tecnologias Assistivas: Ampliadores e leitores de tela,". (2013). Disponível: acessibilidade.w3c.br/pesquisa/resultados-preliminares/. Acesso em: 20 fev 2018.

W.A.I. (WAI), "IndieUI Overview," 26 Junho 2014. Disponível em: http://www.w3.org/WAI/intro/indieui . Acesso: 10 mar 2018.

WCAG, "Web Content Accessibility Guidelines (WCAG) 2.0," (2014). Disponível em: https://www.w3.org/Translations/WCAG20-pt-br/. Acesso em: 15 abr 2018.

Wohlin, C., Runeson, P., Höst, M., Ohlsson, M.C., Regnell, B., Wesslén, A., (2000), Experimentation in Software Engineering: an Introduction, Massachusetts,USA, Kluwer Academic Publishers,0-7923-8682-5. 